\title{
Theory and applications of artificial immune systems
}

\author{
Xiao-Zhi Gao $\cdot$ Mo-Yuen Chow $\cdot$ David Pelta $\cdot$ \\ Jon Timmis
}

Published online: 29 June 2010

(C) Springer-Verlag London Limited 2010

For many years, biology has served as inspiration for the development of novel solutions to computer science and engineering problems. One of the more recent developments in this area is the use of the immune system as inspiration to solve such problems. The area of artificial immune systems (AIS) arose from the inter-disciplinary workings of immunologists and computer scientists to develop solutions to such problems as distributed control and computer security. The field has developed significantly over the recent years, with the development of solutions to a wide variety of problems ranging from document analysis, optimization, dynamic error detection, intrusion detection, robot control and bioinformatics. Within the AIS, there are common, well-understood algorithms, based on specific aspects of immunology. Clonal selection algorithms are the most widely employed and take their inspiration from how cells of the adaptive immune system (specifically, cells called B cell) interact with 'antigen' (something that causes an immune response) and then undergo a process of cloning, mutation and selection. This process has some similarities with the basic

\section{X.-Z. Gao (ه)}

Aalto University, Aalto, Finland

e-mail: gao@cc.hut.fi

\section{M.-Y. Chow}

North Carolina State University, Raleigh, NC, USA

e-mail: chow@ncsu.edu

\section{Pelta}

University of Granada, Granada, Spain

e-mail: dpelta@decsai.ugr.es

\section{J. Timmis}

University of York, York, UK

e-mail: jtimmis@cs.york.ac.uk concept of evolution, and it is no surprise that the algorithms inspired by clonal selection have much in common with well-known evolutionary algorithms and have been applied in a similar manner. Negative selection algorithms, based on how immune cells (known as T cells) are matured in a gland called the thymus, are also very popular. This process allows for the filtering of non-reactive cells in the immune system and has been used as inspiration for building computer security systems, where there is some knowledge of what normal or self is. Finally, immune network algorithms are based on a theory regarding how immune cells (in this case B cells) are maintained as memory in the immune system over time. These B cells interact with each other in a stimulatory and inhibitory way to maintain the memory. This has been used as a metaphor to create dynamic learning algorithms and optimization methods.

The special issue on the AIS is dedicated to the latest work in the theory and applications in this exciting area. Our aim is to provide several case studies of the AIS in solving typical real-world problems. After a detailed review process, a total of three papers were selected for this special issue.

The first paper entitled 'An immune-inspired multiobjective approach to the reconstruction of phylogenetic trees' by Guilherme P. Coelho, Ana Estela A. da Silva, and Fernando J. Von Zuben proposes a new multi-objective optimization technique, namely omni-aiNet, in the reconstruction of phylogenetic trees. This work is based on the immune network metaphor. The phylogenetic trees are often used to represent the relationship among different species, whose construction can be considered as a difficult multi-objective optimization problem of simultaneously minimizing two criteria: minimum evolution and meansquared error. In this paper, the immune algorithm is 
combined with the neighbor-joining method to deal with the phylogenetic reconstruction. Compared with the popular NSGA-II, the paper shows that the proposed hybrid scheme can yield a superior multi-objective optimization performance.

Riff et al. propose an efficient artificial immune algorithm in their paper "A graph-based immune-inspired constraint satisfaction search' to solve constraint satisfaction problems (CSPs). The contributions of this paper are an immune-inspired algorithm guided by the constraints graph, which can solve hard CSPs, and a new application of the tuning method Relevance Estimation and Value Calibration (REVAC) proposed for evolutionary algorithms. The authors term their algorithm "CD-NAIS" which stands for Constraint Directed Network Artificial Immune System. Different tests are carried out using randomly generated binary constraint satisfaction problems and instances of the 3-coloring problem with different constraint networks. CD-NAIS on average has performed $28 \%$ better than SAW (one of the best-known evolutionary algorithms). The calibrated CD-NAIS can solve more problems than GSA, which is a sophisticated genetic algorithm that incorporates many constraint concepts to solve a CSP.
'Immunocomputing for intelligent signal processing' by Alexander O. Tarakanov describes an approach to intelligent signal processing based on immunocomputing (IC). The approach includes both low-level feature extraction and high-level (intelligent) pattern recognition. The key model is the formal immune network (FIN), which includes apoptosis (programmed cell death) and immunization and controls them by cytokines (messenger proteins). The results of computational experiments suggest that the speed and accuracy of the IC approach is superior with other robust methods of computational intelligence and machine learning (in particular, neurocomputing, SVM and evolutionary algorithms).

We would like to thank Prof. John MacIntyre, Editor-inChief of Neural Computing \& Applications, for giving us the opportunity to compile this special issue. We would also like to thank the authors who submitted papers to this issue, and we wish to acknowledge the excellent team of reviewers for their hard work. X. Z. Gao's work was funded by the Academy of Finland under Grants 214144 and 135225. D. Pelta acknowledges the support from the Spanish Ministry of Science and Innovation (project TIN2008-01948), and Consejería de Innovación, Ciencia y Empresa, Junta de Andalucia (project TIC-02970). 